\title{
Efecto del precultivo con sacarosa en la criopreservación por vitrificación de plántulas de Sideritis leucantha Cav. subsp. leucantha producidas in vitro
}

\author{
Effect of sucrose pre-culture on cryopreservation by vitrification of Sideritis leucantha Cav. subsp. \\ leucantha seedlings produced in vitro
}

Fecha de recepción y aceptación: 20 de febrero de 2021 y 17 de marzo de 2021

DOI: https://doi.org/10.46583/nereis_2021.13.820

\author{
Jorge Juan-Vicedo $^{1 *}$ Jorge Eliseo Ramírez-Luna² $^{2}$ Abel Piqueras $^{3}$ y José Luis Casas ${ }^{2}$ \\ ${ }^{1}$ Instituto de Investigación en Medio Ambiente y Ciencia Marina (IMEDMAR). Universidad Católica de Valencia San Vicente Mártir. \\ ${ }^{*}$ Correspondencia: Universidad Católica de Valencia. Instituto de Investigación en Medio Ambiente y Ciencia Marina (IMEDMAR). \\ Calle Guillem de Castro, 94. 46001 Valencia. España.E-mail: jorge.juan@ucv.es \\ ${ }^{2}$ Instituto Universitario de Investigación CIBIO (Centro Iberoamericano de la Biodiversidad), Universidad de Alicante. \\ ${ }^{3}$ Departamento de Mejora Vegetal. Centro de Edafología y Biología Aplicada del Segura (CEBAS-CSIC).
}

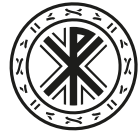

Universidad $\underset{\text { San Vicente Mártir }}{\text { Católica de Valencia }}$

\section{RESUMEN}

Sideritis leucantha Cav. subsp. leucantha es un endemismo valenciano con uso medicinal que tiene diversos problemas de conservación. Por tanto, el objetivo principal de este trabajo fue probar el efecto de un tratamiento de precultivo para la posterior criopreservación de brotes apicales de esta especie. Los resultados mostraron que el precultivo con sacarosa $0.3 \mathrm{M}$ durante 24 horas resultó en una mayor supervivencia de los explantes y regeneración in vitro después de tres tratamientos de vitrificación ensayados. Estos resultados sugieren que el acondicionamiento de los explantes puede mejorar la supervivencia de los explantes de S. leucantha subps. leucantha cultivados in vitro tras la criopreservación.

PALABRAS CLAVE: criopreservación, cultivo in vitro, plantas endémicas, plantas medicinales, precultivo.

\section{ABSTRACT}

Sideritis leucantha Cav. subsp. leucantha is a Valencian endemic medicinal plant that faces some conservation constraints. Therefore, this work aimed to test a preconditioning treatment for the further cryopreservation of shoot tip explants of this species. The results showed that pre-culture in sucrose $0.3 \mathrm{M}$ for 24 hours resulted in the highest explant survival and regeneration after three vitrification approaches. These findings point out that preconditioning of explants may enhance cryopreservation performance in tissue cultured plantlets of $S$. leucantha subps. leucantha.

KEYWORDS: cryopreservation, endemic plants, in vitro culture, medicinal plants, preconditioning. 


\section{INTRODUCCIÓN}

El género Sideritis está constituido por alrededor de 150 taxones silvestres de distribución circunmediterránea [1]. Son plantas aromáticas que se han utilizado en medicina popular [2-4] por las propiedades antiinflamatorias, antimicrobianas, antisépticas y antioxidantes de su aceite esencial [5-9]. Sideritis leucantha Cav. subsp. leucantha es un endemismo valenciano que se emplea en medicina tradicional como antiinflamatorio respiratorio, tónico estomacal y digestivo, antihemorroidal y vulnerario [4]. Aunque no presenta problemas graves de conservación se han detectado algunas amenazas como la presión urbanística, la transformación agrícola del territorio donde crece y la recolección incontrolada que están mermando las poblaciones naturales y hacen necesario reforzar las estrategias de conservación in situ y ex situ para esta especie [4, 10, 11].

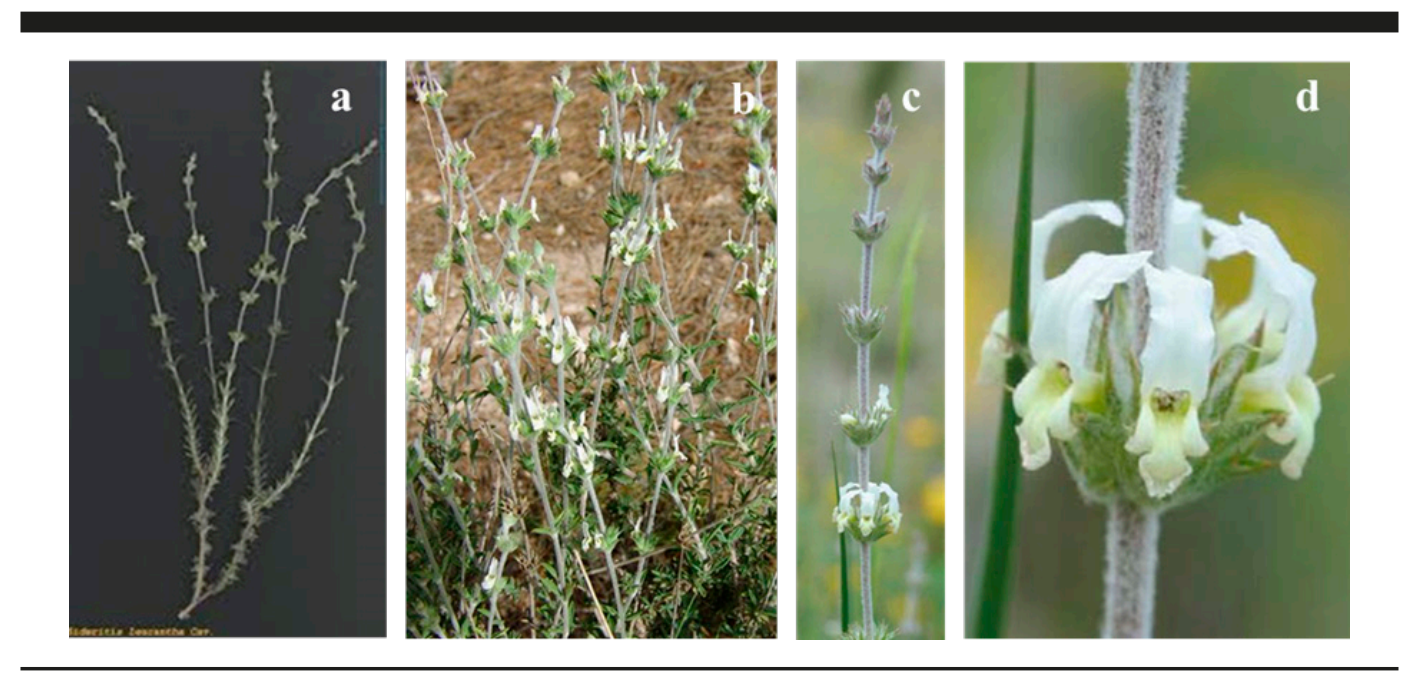

Fig. 1. Imágenes de Sideritis leucantha Cav. susbsp. leucantha a) Imagen de herbario de la parte área; b) aspecto general de la planta en floración en el ambiente natural donde vive (fuente: Herbari Virtual del Mediterràni Occidental: http://herbarivirtaul.uib.es); c) detalle de un tallo florífero y d) Detalle de un verticilastro de flores en el que se aprecia el indumento lanoso del tallo y las corolas bicolóreas (fuente: Reserva Hyla: http://www.reserva.hyla.es/).

La criopreservación es una herramienta biotecnológica empleada en las estrategias de conservación ex situ de plantas silvestres y cultivares agrícolas $[12,13]$. Se basa en la eliminación de la mayor parte del agua congelable de los tejidos vegetales, lo que permite la inmersión directa de las células y tejidos vegetales en nitrógeno líquido (NL) [14]. La vitrificación es una técnica ampliamente utilizada para la crioconservación de meristemos y ápices de tallos producidos in vitro ya que es relativamente sencilla y económica, requiere poco espacio para el almacenamiento del material vegetal preservado, exige un bajo esfuerzo para su mantenimiento en el largo plazo y reduce los desórdenes fisiológicos y la variación somaclonal en comparación con otras técnicas [14-19]. De hecho, la vitrificación se ha aplicado de forma exitosa en más de 160 especies silvestres y cultivares agrícolas y ha asegurado la supervivencia de los brotes en especies donde otras técnicas de crioconservación habían fallado previamente $[15,20]$. Sin embargo, una de las principales limitaciones de esta técnica es la posible 
toxicidad química y el estrés osmótico producido por los compuestos empleados en la solución vitrificante que pueden dañar de forma irreversible los centros meristemáticos de los tejidos y órganos sometidos a criopreservación [21-24].

Para evitar este problema, algunos autores sugieren la aplicación de una fase de precultivo con sacarosa o polialcoholes como el sorbitol que pueden mejorar significativamente la supervivencia de los explantes crioconservados tras la deshidratación con la solución de vitrificación [20, 25-28].

Por ello, el principal objetivo de este trabajo es estudiar el efecto del precultivo a distintas concentraciones de sacarosa combinado con tres procedimientos distintos de criopreservación en la supervivencia de plántulas de Sideritis leucantha Cav. subsp. leucantha producidas in vitro, como base para el desarrollo de una estrategia integrada de conservación ex situ.

\section{MATERIAL Y MÉTODOS}

\section{Precultivo del material vegetal}

Explantes de 0,1-0,2 cm de longitud con 1 o 2 primordios foliares fueron obtenidos a partir de brotes apicales o axilares de las plantas cultivadas in vitro durante 4 semanas. A todos ellos, se les aplicó un tratamiento de precultivo de 24 horas con sacarosa al $2 \%$. Posteriormente, los explantes se sometieron a concentraciones de sacarosa de 0,3, 0,5 y 0,7 M durante 24 horas más. Los tratamientos de precultivo se desarrollaron a $22{ }^{\circ} \mathrm{C}$ en condiciones de oscuridad.

\section{Tratamiento de vitrificación}

Los explantes precultivados en sacarosa se dividieron en cinco grupos y la vitrificación se llevó a cabo en crioviales de polipropileno de 12,5 x $92 \mathrm{~mm}$ mediante la aplicación de distintas soluciones crioprotectoras y vitrificadoras, previas a la inmersión en NL, siguiendo tres procedimientos distintos de acuerdo con [29] para el Grupo 2, el trabajo de [30] para el Grupo 3 y [28] para el Grupo 4 (tabla 1). Los grupos restantes sirvieron como controles e incluían aquellos explantes que solo habían estado sometidos a un $2 \%$ de sacarosa durante 24 horas, sin precultivo adicional. De este modo, unos explantes fueron sometidos a vitrificación seguida de inmersión en NL (Grupo 1) y otros explantes se sometieron a vitrificación pero no a inmersión en NL en el Grupo 5. Los tratamientos de vitrificación se desarrollaron a $22{ }^{\circ} \mathrm{C}$ y la inmersión en NL duró 1 hora.

\section{Tratamiento de descongelación y descarga}

Los explantes de todos los grupos crioconservados se retiraron del NL y descongelaron en agua destilada estéril a $40^{\circ} \mathrm{C}$ durante 1 minuto. Para su recuperación se pasaron a una solución de sacarosa 1,2 M durante 20 minutos para diluir la mezcla vitrificadora (PVS2 o EG + Sorbitol + BSA). 


\section{Restauración del crecimiento vegetal}

Todos los explantes se cultivaron en medio Murashige y Skoog [31] suplementado con $30 \mathrm{~g} / \mathrm{L}$ de sacarosa y solidificado con Plant Agar (Duchefa), sin reguladores del crecimiento. Los cultivos se incubaron a $22 \pm 3{ }^{\circ} \mathrm{C}$ en condiciones de oscuridad durante 2 días. Posteriormente, se sometieron a un fotoperiodo 16 horas-luz, con una intensidad lumínica de $50 \mu \mathrm{mol} \mathrm{m}-2 \mathrm{~s}-1$ durante 4 semanas más. La supervivencia se tomó como el porcentaje del número total de explantes que restauraron el crecimiento normal después de 4 semanas de cultivo en las condiciones anteriormente descritas.

\section{Diseño experimental y tratamiento estadístico de los datos}

Para cada tratamiento se hicieron cuatro réplicas de 5 explantes cada una y los experimentos se realizaron por triplicado. Los resultados se expresan como medias y sus errores estándar asociados para cada uno de los tratamientos. Para determinar diferencias significativas entre ellos se llevó a cabo un análisis de la varianza de un factor (ANOVA) y las diferencias entre las medias se evaluaron mediante el test de Duncan (Duncan's Multiple Range Test) con un nivel de significancia del $5 \%$. Los porcentajes de plantas que restauraron el crecimiento se ajustaron mediante la transformación raíz cuadrada del arcoseno. Para realizar el análisis de datos se empleó el software libre Infostat 2008 versión estudiantil (https://www.infostat.com.ar/index.php?mod=page\&id=37).

Tabla 1. Resumen de los tres procedimientos de criopreservación estudiados en explantes de Sideritis leucantha Cav. susbsp. leucantha basados en los trabajos previos de Langis [29], Benson [30] y Pennycooke

[28]. En todos los casos, el tiempo de exposición a la solución de carga fue de 20 minutos. El tiempo de inmersión en nitrógeno líquido (NL) tras la exposición a la solución vitrificante fue de 60 minutos en todos los experimentos. SA: sacarosa, GLI: glicerol, EG: etilenglicol, DMSO: dimetil sulfóxido, MS: Murashige y Skoog [31], PVS2: solución de vitrificación para vegetales 2 [(3,26 M GLI, 15 \%, 2,42 M EG, 1,92 M DMSO y sacarosa 0,4 M de acuerdo con (Sakai et al., 1990)], SB: sorbitol, BSA: albúmina de suero bovino

\begin{tabular}{cccc}
\hline Protocolo & $\begin{array}{c}\text { Composición de la } \\
\text { solución de carga }\end{array}$ & $\begin{array}{c}\text { Composición de la } \\
\text { solución vitrificante }\end{array}$ & $\begin{array}{c}\text { Exposición } \\
\text { (min) }\end{array}$ \\
\hline$[29]$ & $1,29 M \mathrm{EG} / 1.29 M \mathrm{DMSO}$ & $\begin{array}{c}8,05 M \mathrm{EG}+10,82 M \\
\mathrm{SB}+0,9 \mathrm{~m} M \mathrm{BSA}\end{array}$ & 60 \\
{$[30]$} & $0,4 M \mathrm{SA}+2 M \mathrm{GLI}+\mathrm{MS}$ & $\mathrm{PVS} 2$ & 60 \\
{$[28]$} & $0,4 M \mathrm{SA}+2 M \mathrm{GLI}+\mathrm{MS}$ & $\mathrm{PVS} 2$ & 30 \\
\hline
\end{tabular}

\section{RESULTADOS}

Los resultados obtenidos mostraron que el precultivo con sacarosa $0,3 \mathrm{M}$ durante 24 horas indujo una supervivencia significativamente mayor a la obtenida con la exposición a $0,5 \mathrm{M}$ o $0,7 \mathrm{M}$ de sacarosa en los tres protocolos de crioconservación ensayados, muy cercanos a los porcentajes de supervivencia del grupo control (tabla 2). En lo que al protocolo de crioconservación respecta, las mayores tasas de regeneración de explantes se produjeron en el Grupo 4, basado en el procedimiento descrito en [28], para las tres concentraciones de sacarosa ensayadas $(0,3,0,5$ y $0,7 \mathrm{M})$. 
Tabla 2. Efecto del precultivo a distintas concentraciones de sacarosa, seguido de tratamiento de criopreservación, en la supervivencia de explantes de Sideritis leucantha Cav. subsp. leucantha producidos in vitro. La supervivencia se ha evaluado como el porcentaje de explantes que han mostrado signos de crecimiento (elongación del tallo y desarrollo foliar) tras 8 semanas de cultivo in vitro. Los datos se expresan como medias \pm error estándar; superíndices con letras minúsculas distintas indican diferencias significativas sobre

la base del test de Duncan (Duncan's Multiple Range Test) con un nivel de significancia del $5 \%$

\begin{tabular}{cccccc}
\hline \multirow{2}{*}{ Tratamiento de criopreservación } & \multicolumn{5}{c}{ Concentración de sacarosa (M) } \\
& 0,3 & 0,5 & 0,7 & Control & \\
\hline$[29]$ & $6,6 \pm 6^{\mathrm{d}}$ & $13,3 \pm 6^{\mathrm{d}}$ & $6,6 \pm 6^{\mathrm{d}}$ & $86,6 \pm 8^{\mathrm{a}}$ & Crecimiento \\
{$[30]$} & $53,3 \pm 13^{\mathrm{bc}}$ & $40,0 \pm 12^{\mathrm{c}}$ & $40,0 \pm 8^{\mathrm{c}}$ & $93,3 \pm 6^{\mathrm{a}}$ & $\begin{array}{c}\text { vegetal } \\
(\% \pm \mathrm{SE})\end{array}$ \\
{$[28]$} & $73,3 \pm 6^{\mathrm{ab}}$ & $46,6 \pm 8^{\mathrm{c}}$ & $53,3 \pm 13^{\mathrm{bc}}$ & $93,3 \pm 6^{\mathrm{a}}$ & \\
\hline
\end{tabular}

\section{DISCUSIÓN}

El proceso completo de crioconservación por vitrificación consta, en esencia, de tres fases que persiguen la aclimatación fisiológica de los explantes a la fase criogénica (enfriamiento) mediante la aplicación de sustancias crioprotectoras, el enfriamiento con NL y la posterior recuperación del material vegetal. Sin embargo, este proceso experimental puede dañar los explantes tratados debido a la toxicidad y presión osmótica generados por los componentes empleados [21-24].

Para intentar superar estas limitaciones inherentes a la vitrificación, se ha sometido a los explantes a un tratamiento de precultivo con sacarosa a $0,3,0,5$ y $0,7 \mathrm{M}$ previo a la criopreservación por tres métodos descritos en la literatura científica [28-30].

En el presente trabajo, las mayores tasas de regeneración $(73,3 \pm 6 \%)$ se obtuvieron con el precultivo en sacarosa $0,3 \mathrm{M}$ durante 24 horas en aquellos explantes crioconservados mediante el protocolo [28]. Resultados similares se han obtenido en especies silvestres del género Dioscorea [32] y Limonium [33] o cultivares de rosa [27], clavel [34,35], batata [28] o uva [26].

Valores intermedios de supervivencia (45-55\%) fueron obtenidos para el mismo protocolo en el resto de las concentraciones de sacarosa, o con el protocolo [30] a una concentración de sacarosa de $0,3 \mathrm{M}$. El resto de los tratamientos indujeron una menor supervivencia, siendo especialmente significativa la mortalidad en aquellos explantes crioconservados siguiendo el procedimiento expuesto en [29] para las tres concentraciones de sacarosa empleadas en el precultivo.

Los resultados obtenidos en el presente trabajo sugieren, en primer lugar, que los explantes precultivados en sacarosa $0,3 \mathrm{M}$ durante $24 \mathrm{~h}$ adquieren suficiente osmotolerancia a las soluciones vitrificantes (como PVS2) siguiendo los protocolos de crioconservación propuestos en [28, 30]. Además, teniendo en cuenta las variables de concentración de sacarosa y el método de criopreservación, también se puede deducir que el precultivo en sacarosa actúa de forma sinérgica con otros componentes de la solución de carga tales como el glicerol. Este compuesto podría ofrecer una osmotolerancia adicional que, asociada a los menores tiempos de exposición al PVS2 en los explantes del Grupo 4 
(procedimiento basado en [28]) muestra las mayores tasas de supervivencia para todas las concentraciones de sacarosa ensayadas (tabla 2). Hirai y Sakai [20, 25] también obtuvieron resultados similares con mezclas de glicerol $2 \mathrm{M}$ y varias concentraciones de sacarosa, con tasas más altas de formación de tallos que los meristemos tratados solo con sacarosa. En este sentido, [24, 36] también comprobaron, además, que hay un mayor porcentaje de supervivencia a menores tiempos de exposición a la solución PVS2, tal y como se ha observado en este trabajo para los explantes de S. leucantha subsp. leucantha. Esto podría deberse a una menor penetración en la célula vegetal de compuestos presente en la solución de vitrificación que pueden ejercer presión osmótica y toxicidad.

\section{CONCLUSIONES}

El precultivo de explantes de $S$. leucantha subsp. leucantha con sacarosa 0,3 M durante 24 horas resultó en una mayor supervivencia del material vegetal tras los tratamientos de criopreservación aplicados. Las mayores tasas de regeneración se dieron con precultivo en sacarosa $0,3 \mathrm{M}$ seguido del tratamiento con la solución de carga compuesta por glicerol $2 \mathrm{M}$ y sacarosa $0,4 \mathrm{M}$, previo a la exposición a la solución vitrificante PVS2 durante 30 minutos. Estos resultados muestran, en conjunto, niveles satisfactorios de acondicionamiento y regeneración de explantes de S. leucantha subsp. leucantha. Los resultados obtenidos en el presente trabajo suponen la base para el establecimiento de estrategias de conservación a largo plazo de la especie basadas en la criopreservación de material vegetal producido in vitro.

\section{AGRADECIMIENTOS}

Este trabajo ha sido posible gracias al proyecto de la Generalitat Valenciana GV04B-459. Además, J. E. R. L. obtuvo una beca de la Universidad de Alicante para la formación de doctores.

\section{REFERENCIAS BIBLIOGRÁFICAS}

[1] Barber JC, Santos-Guerra A, Turner KG, Jansen RK. Origin of Macaronesian Sideritis L. (Lamioideae: Lamiaceae) inferred from nuclear and chloroplast sequence datasets. Mol. Phylogenet. Evol. 2002;23(3):293-306.

[2] Font Quer P. Plantas Medicinales. Barcelona: Ed. Labor; 1962.

[3] González-Burgos E, Carretero ME, Gómez-Serranillos MP. Sideritis spp.: Uses, chemical composition and pharmacological activities. A review. J Ethnopharmacol. 2011;35:209-225.

[4] Pardo de Santayana M, Morales R, Tardío J, Molina M. Inventario español de los conocimientos tradicionales relativos a la biodiversidad. Fase II. Madrid: Ministerio de Agricultura y Pesca, Alimentación y Medio Ambiente; 2018.

[5] Barberán FAT, Tomás F, Ferreres. Two flavone glycosides from Sideritis leucantha. Phytochemistry. 1984;9:2112-2113. 
[6] Barberan FAT, Mañez S, Villar. Identification of antiinflammatory agents from Sideritis species growing in Spain. J Nat Prod. 1987;50:313-314.

[7] Iscan G, Kirimer N, Kurkcuoglu M, Baser KHC. Composition and antimicrobial activity of the essential oils of two endemic species from Turkey: Sideritis cilicica and Sideritis bilgerana. Chem Nat Comp. 2005;41:679-682.

[8] Dulger B, Gonuz A, Aysel V. Inhibition of clotrimazole-resistant Candida albicans by some endemic Sideritis species from Turkey. Fitoterapia. 2006;77:404-405.

[9] Tsaknis J, Lalas S. Extraction and identification of natural antioxidant from Sideritis euboea (mountain tea). J Agric Food Chem. 2005;53:6375-6381.

[10] Laguna E. Flora rara, endémica o amenazada de la Comunidad Valenciana. Valencia: Generalitat Valenciana, Conselleria de Medio Ambiente; 1998.

[11] Allen DJ. Sideritis leucantha. The IUCN Red List of Threatened Species; 2014: e.T203269A2762702. Disponible en: http://dx.doi.org/10.2305/IUCN.UK.20141.RLTS. T203269A2762702.en

[12] Reed BM. Plant Cryopreservation: A Practical Guide. New York: Springer-Verlag; 2008.

[13] González-Benito ME, Martín C. In vitro preservation of Spanish biodiversity. In Vitro Cell Dev Biol Plant. 2011;47:46-54.

[14] Charoensub R, Phansiria S, Yongmanitchai W, Sakai A. Routine cryopreservation of in vitrogrown axillary apices of cassava (Manihot esculenta Crantz) by vitrification: importance of a simple mononodal culture. Sci Horticult. 2003;98:485-492.

[15] Towill LE. Cryopreservation by vitrification. En: B. Grout, editor. Genetic Preservation of plant cells in vitro. New York: Springer Verlag; 1995.

[16] Channuntapipat C, Collins G, Sedgley M. Conservation of almond germplasm by cryopreservation. Cahiers Options Méditerranéennes. 2001;56:101-106.

[17] Takagi H, Tien Thinh N, Islam OM, Senboku T, Sakai A. Cryopreservation of in vitro-grown shoot tips of taro (Colocasia esculenta (L.) Schott) by vitrification. 1. Investigation of basic conditions of the vitrification procedure. Plant Cell Rep. 1997;16:594-599.

[18] Panis B, Lambardi M. Status of cryopreservation technologies in plants (crops and forest trees). The role of biotechnology. Villa Gualino, Turín, Italia - 5-7 marzo; 2005.

[19] Engelmann F. Plant cryopreservation: Progress and prospects. In vitro Cell. \& Dev. Biol. Plant. 2004;40:427-433.

[20] Hirai D, Sakai A. Cryopreservation of in vitro-grown axillary shoot-tip meristems of mint (Mentha spicata L.) by encapsulation vitrification. Plant Cell Rep. 1998;19:150-155.

[21] Luo J, Reed BM. Abscisic Acid-Responsive Protein, Bovine Serum Albumin, and Proline Pretreatments Improve Recovery of in Vitro Currant Shoot-Tip Meristems and Callus Cryopreserved by Vitrification. CryoBiology. 1997;34:240-250.

[22] Efendi D. Transformation and cryopreservation of embryogenic avocado (Persea americana Mill.) cultures [tesis doctoral]. University of Florida; 2003.

[23] Lambardi M, Fabbri A, Caccavale A. Cryopreservation of white poplar (Populus alba L.) by vitrification of in vitro-grown shoot tips. Plant Cell Rep. 2000;19:213-218.

[24] Volk GM, Walters C. Plant vitrification solution 2 lowers water content and alters freezing behavior in shoot tips during cryoprotection. Cryobiology. 2006;52(1):48-61. 
[25] Hirai D, Sakai A. Simplified cryopreservation of sweet potato [Ipomoea batatas (L.) Lam.] by optimizing conditions for osmoprotection. Plant Cell Rep. 2003;21:961-966.

[26] Matsumoto T, Sakai A. Cryopreservation of axillary shoot tips of in vitro-grown grape (Vitis) by a two-step vitrification protocol. Euphytica. 2003;131:299-304.

[27] Halmagyi A, Pinker I. Plant regeneration from Rosa shoot tips cryopreserved by a combined droplet vitrification method. Plant Cell Tissue Organ Cult. 2006;84:145-153.

[28] Pennycooke JC, Towill LE. Cryopreservation of shoot tips from in vitro plants of sweet potato [Ipomoea batatas (L.) Lam.] by vitrification. Plant Cell Rep. 2000;19:733-737.

[29] Langis R., Schneibel B, Earle ED, Steponkus PL. Cryopreservation of carnation shoot tips by vitrification. Cryobiology. 1990;27:657-658.

[30] Benson EE. Cryopreservation. Plant cell culture. A practical approach. 2. ${ }^{a}$ ed. London: Oxford University Press; 1996.

[31] Murashige T, Skoog F. A revised medium for rapid growth and bio assays with tobacco tissue cultures. Plant Physiol. 1962;15:473-497.

[32] Ahuja S, Mandal BB, Dixit S, Srivastava PS. Molecular, phenotypic and biosynthetic stability in Dioscorea floribunda plants derived from cryopreserved shoot tips. Plant Sci. 2002;163:971977.

[33] Matsumoto T, Takahashi C, Sakai A, Nako Y. Cryopreservation of in vitro-grown apical meristemos of hybrid statice by three different procedures. Scientia Horticult. 1998;76:105-114.

[34] Tannoury M, Ralabosoa J, Kaminski M, Dereuddre. Cryoconservation par vitrification d'apex enrobés d'œillet (Dianthus caryophyllus L.) cultivé in vitro. C. R. Acad. Sci. Paris. 1991;313:633638.

[35] Tannoury M, Vintéjoux C, Dereuddre. Cryoconservation par encapsulation et déhydratation d'apex d'œillet (Dianthus caryophyllus L.) cultivés in vitro. C. Acta bot. Gallica. 1995;142(5):415424.

[36] Volk GM, Caspersen AM. Plasmolysis and recovery of different cell types in cryoprotected shoot tips of Mentha x piperita. Protoplasma. 2007;231:215-226. 